\title{
Session 7: Molecular biology (III)
}

Tuesday 17 September 2002, Moderators: O.R. Burrone and R. Burioni

\section{[16.20-16.40]}

\section{Cloning and Expression of a non-small cell lung cancer specific RM2 scfv in Pichia pastoris P. Watchmaker, M. Rani, K. Kota, S. Chakrabarti and S. Mukerjee \\ Shantha Biotechnics Pvt Ltd, Hyderabad, India}

Single-chain variable fragments (scFvs) are small tumor-recognition units that hold enormous potential in antibody-based therapeutics. Advantages of scfv over whole antibodies are now explored for radioimmunodetection and for insitu radiotherapy of cancer due to potentially better tumor penetration and blood clearance and reduced immunogenicity. Their clinical applications, however, require the large scale production and purification of biologically active recombinant scFvs. RM2 IgG1.k is a human monoclonal antibody recognizing melanoma, pancreatic and nonsmall cell lung carcinoma. The variable light (VL) and variable heavy $(\mathrm{VH})$ chain domains which form the antigen binding site for the RM2 Mab were rescued using RT-PCR and expressed as a monomeric scfv fragment in which the VL and the VH domains are connected by a (Gly4Ser)3 linker. scfv derived were constructed in both orientations, i.e., Vh-linker-Vl and Vllinker-Vh, but only the latter form could be expressed and secreted in the recombinant Pichia pastoris system. The vectors were derived from plasmid pPIC9 (Invitrogen) where the expression cassette is under the control of the strong AOX I promoter and downstream of the alpha-mating signal sequence. For proper processing and expression of the RM2 scfv gene the vector was digested with $\mathrm{XhoI} / \mathrm{SnaB} 1$ and KEX2 site recreated by the addition of glu-lys-arg at the 3' end of VL using oligonucleotide GTCTCGAGAAAAGATCCTATGAGC. The RM2 scfv was also cloned and expressed as his-tag fusion protein in E.coli expression vector Pet24a. For the removal of extra gene sequences the vector was digested with $\mathrm{NdeI} / \mathrm{SalI}$ and directional cloning of the gene was done at HindIII/XhoI site. Both the Pichia expressed scfv and E.coli expressed scfv- histag was analyzed for purity and immunoreactivity by western blotting, FACS, and competitive ELISA. Western blotting and FACS was done using a mouse anti-linker Mab which clearly showed that as the parent antibody, the RM2scfv and RM2scfv-histag recognized a $52 \mathrm{kD}$ ImSF antigen expressed on the surface of live melanoma, pancreatic and non- small cell lung carcinoma cells. The expression of scFvs in P. pastoris was 30 to 40-fold higher than in Escherichia coli. Protein expression, culture conditions, and purification were optimized in 1-L cultures. The large-scale production of RM2 scfv in a $10 \mathrm{~L}$ fermenter gave rough yields of about $1 \mathrm{~g} / \mathrm{L}$. The $\mathrm{scFv}$ antibody was purified using a hydrophobic column which gave good yields and purity. Biodistribution studies in athymic mice bearing human carcinoma xenografts showed tumortargeting of RM2 scfv. In conclusion,improved yields of monovalent scFvs were achieved using the P. pastoris expression/secretion system. The in vitro and in vivo properties of these scFvs suggest specific tumor targeting activity thereby indicating possible therapeutic applications.

\section{[16.40-17.00]}

Human recombinant autoantibodies specific for the La (SS-B) autoantigen from patient derived phage display combinatorial antibody libraries J.M.H. Raats, W.F. Roeffen, A.J.C.M. van Sluisveld, S. Litjens, I. Bulduk, G. Mans, F.H.J. Van den Hoogen, G.J.M. Pruijn and W.J. van Venrooij University of Nymegen, The Netherlands

In many patients with autoimmune disorders, protein antigens associated with RNA in ribonucleoprotein particles (RNPs) become targets of the immune system leading to the generation of autoantibodies. Some of such autoantibodies are highly specific for a particular autoimmune disease. For instance autoantibodies against components of the Ro RNP particles are found in patients with systemic lupus erythematosus (SLE) 
or systemic sclerosis (SS). Ro RNPs are composed of several proteins complexed with the small cytoplasmic hY RNA's. Each human Ro-RNP consists of one of the four hY RNA's with their associated proteins Ro-60 and La (SSB). Phopshorylation of serine 366 in the La protein plays an important role. Dephosphorylated $\mathrm{La}$ is transcriptionally active, whereas dephosphorylated La is inactive. It was recently shown that La is efficiently dephosphorylated during apoptosis. This observation was made after induction of apoptosis by various initiators and in various cell types. It was also shown that at least a subset of the La protein is proteolitically cleaved in vivo, generating a $45 \mathrm{kDa}$ frag.

In this paper we explore the autoantibody repertoire present in SLE and SS autoimmune patients directed against the La protein. By panning SLE and SS patient derived phage display antibody libraries against both recombinant La protein and the phosphopeptide P366 derived thereof, we were able to isolate a large number of different La specific antibody fragments. All clones were sequenced and tested for La specificity by ELISA, LIA and western blotting. La epitope specificities of the individual clones were analysed. Finally, the individual anti-La antibodies were tested for their recognition of apoptotically modified La protein and the localisation of the different isoforms of La within the cell was studied.

\section{[17.00-17.20]}

\section{Production and characterization of monoclonal antibodies produced in the human cell line \\ PER.C6.}

David Jones, Nathalie Kroos, Regina Anema, Nelie van Dijk, Hans Kapteyn, Renate Zwijsen, Ton Logtenberg and Abraham Bout

Crucell, Archimedesweg 4, 2301 CA Leiden, The Netherlands

The production of human monoclonal antibodies with high bioactivity in stable, large scale batches presents a number of manufacturing system challenges. We have developed a monoclonal antibody manufacturing system that not only produces high yields of antibody in scalable, serum-free culture conditions, but also creates human glycosylation structures and optimizes bioactivity.

We have produced several different fully human monoclonal antibodies in PER.C6, a well-characterized human cell line which can grow as adherent cells and also in serum-free suspension. The cells were transfected with a vector containing the heavy and light chains of our antibody, several hundred colonies were picked, and 35 high-producing clones were selected for characterization. The highest expressing clones were able to produce in excess of $50 \mathrm{pg} / \mathrm{seeded}$ cell/day of antibody.

We evaluated stability, glycosylation, robustness of growth in suspension media, and bioactivity of antibody produced in PER.C6. Clones were tested for stability of expression and exhibited stable antibody expression over a 4-week period in the absence of selection pressure. Glycoanalysis reveals that the have glycosylation similar to that seen on $\mathrm{IgG}$ in human serum. Furthermore, our initial analysis shows a correlation between the glycosylation of the antibody and the bioactivity (determined by CDC).

Our initial data indicate that PER.C6 can produce high concentrations of monoclonal antibody where the glycosylation can be manipulated and bioactivity influenced by the media conditions. Additionally, we are developing a process that would enable large scale batches to be manufactured under serum-free culture conditions and aggressive timelines.

[17.20-17.40]

Biological activity of human "conditional" anti-Fc $\varepsilon R I \alpha$-chain antibodies is defined by their light chain

M. Fux ${ }^{\mathrm{a}}$, T. Bobrzynski ${ }^{\mathrm{a}}$, M. Vogel ${ }^{\mathrm{a}}$, J. Pachlopnik $^{\mathrm{a}}$, M. Horn ${ }^{\mathrm{c}}$, B.M. Stadler ${ }^{\mathrm{a}}$ and S.M. Miescher ${ }^{\mathrm{a}, \mathrm{b}}$

anstitute of Immunology, Inselspital, Bern,

Switzerland

${ }^{\mathrm{b}}$ ZLB Bioplasma AG, Bern, Switzerland

${ }^{\mathrm{c}}$ Berna Biotech Ltd, Bern, Switzerland

Autoantibodies reacting with the $\alpha$-chain of the human high affinity $\operatorname{IgE}$ receptor $(\mathrm{Fc} \varepsilon \mathrm{RI} \alpha)$ have been implicated in autoimmune urticaria. We have recently isolated a range of different anti $\alpha$-chain antibodies from phage libraries constructed from urticaria (UM $\alpha 16, \mathrm{UG} \alpha 8$ ) and tonsils of healthy donors (LTM $\alpha 15)$. UM $\alpha 16$ and $\operatorname{LTM} \alpha 15$ originate from IgM libraries and UG $\alpha 8$ from an IgG library. We designated these antibodies as conditional antibodies because they recognise the $\alpha$-chain of Fc $\varepsilon$ RI only if the receptor is not occupied by IgE. Produced as full length antibodies in HEK cells UM $\alpha 16$ and LTM $\alpha 15$ showed strong recognition of the $\alpha$-chain whereas $\mathrm{UG} \alpha 8$ showed a weaker reaction and was not anaphylactogenic. DNA sequencing revealed that both IgM antibodies have identical heavy and light chain sequences. They differ from the UG $\alpha 8$ by three amino acids in framework 4 
of the heavy chain. Also, $\mathrm{UM} \alpha 16$ and $\operatorname{LTM} \alpha 15$ have a completely different light chain compared to the UG $\alpha 8$.

To investigate the contribution of the light chain for recognition of the Fc $\varepsilon$ RI $\alpha$-chain, we combined the $\operatorname{IgM}$ heavy chain with the $\operatorname{IgG}$ derived light chain and vice versa. Using these shuffled antibodies, we demonstrated that the light chain originally associated with IgM was necessary for high affinity recognition of the $\alpha$-chain.

Our results indicate that human natural anti-Fc $\varepsilon \mathrm{RI} \alpha-$ chain antibodies which can function as conditional antibodies show a restricted repertoire and can be isolated from the $\operatorname{IgM}$ repertoire of both healthy donors and urticaria patients.

\section{[17.40-18.00]}

\section{Human miniantibodies against orthopoxviruses}

A.A. Ilyichev, V.V. Morozova, N.V. Tikunova, E.F. Belanov, A.A. Guskov, N.I. Bormotov, T.A. Batanova, E.I. Bovshik, E.V. Jirakovskaja, O.A. Serova, T.E. Yun and L.S. Sandakhchiev State Research Center of Virology and Biotechnology VECTOR, Koltsovo, Novosibirsk region, 630559 Russia

Forty five human recombinant monoclonal antibodies against vaccinia virus, Elstree, L-IVP variant, have been selected from a combinatorial phage library of human single-chain antibody fragments, scFv, (Medical Research Council Centre, Cambridge, England) using biopanning procedure. Affinity of the selected antibodies was assayed using indirect ELISA with subsequent dilutions of both vaccinia virus and the recombinant antibodies. Obtained antibodies were shown to titer until $10^{14}-10^{15} \mathrm{~mol} / \mathrm{ml}$, and minimal amount of detected vaccinia virus is $150 \mathrm{ng} / \mathrm{ml}$. Epitopes of the surface proteins of orthopoxviruses were analyzed with the aid of the selected recombinant antibodies. In order to find difference in the epitopes, binding of selected antibodies was assayed with the different orthopoxviruses: cowpox, rabbitpox, monkeypox, ectromelia viruses and smallpox viruses (India 3A, Butler, Kuwait, etc). In addition, selected antibodies were tested for their neutralizing activity with orthopoxviruses (smallpox, cowpox, rabbitpox, monkeypox, ectromelia viruses and vaccinia virus) in plaque reduction neutralization test using VeroE6 monolayer.
[18.00-18.20]

Using XenoMouse ${ }^{\circledR}$ Mice for generating optimal human antibodies for therapeutic leads

Larry L. Green

Abgenix, Inc., 6701 Kaiser Drive, Fremont, CA 94555, USA

An optimal therapeutic antibody almost always must do more than simply bind to its antigen target. A project to generate a therapeutic antibody would include plans for its mechanism(s) of action, potency, and affinity. The design goals might include qualitative and quantitative criteria for antagonist or agonist activity, affinity, internalization, and effector functions, in addition to the antibody having a minimal chance of being immunogenic in humans. Having a large pool of quality fully human antibodies from which to identify the best antibody candidates is key to achieving the design goals of a program.

Abgenix's XenoMouse ${ }^{\circledR}$ mice are strains of mice genetically engineered to produce quality fully human $\operatorname{IgG} \kappa$ and $\operatorname{IgG} \lambda$ monoclonal antibodies. Using either hybridoma technology or Abgenix's proprietary XenoMax ${ }^{\mathrm{TM}}$ technology, human monoclonal antibodies with therapeutic-grade affinity and potency are recovered directly from XenoMouse mice, consequently eliminating the need for antibody optimization through molecular engineering. Abgenix's hybridoma process typically yields many dozens to hundreds of antigen-specific antibodies for subsequent characterization. The XenoMax process directly interrogates the fully human antibody repertoire of hyperimmunized XenoMouse animals, typically identifying hundreds to thousands of antigen-specific antibodies and thereby enabling the recovery of antibodies with very rare characteristics. The Abgenix hybridoma and XenoMax processes for antibody generation complement each other, increasing the probability of a timely capture of the best antibodies that meet the design goals. At Abgenix, these technologies are integrated into a larger workflow of screening and target validation to advance the product discovery process. This workflow includes upfront functional testing, rapid biophysical characterization, pre-clinical validation and integrated process sciences and manufacturing. This product discovery engine accelerates the advancement of optimal therapeutic leads into clinical trials. 